\title{
On the Harmonic Index of Triangle-Free Graphs*
}

\author{
Jianxi Liu \\ Department of Applied Mathematics, School of Informatics, Guangdong University of Foreign Studies, Guangzhou, China \\ Email: liujianxi2001@gmail.com, ljx@oamail.gdufs.edu.cn
}

Received June 7, 2013; revised July 7, 2013; accepted July 14, 2013

Copyright (C) 2013 Jianxi Liu. This is an open access article distributed under the Creative Commons Attribution License, which permits unrestricted use, distribution, and reproduction in any medium, provided the original work is properly cited.

\begin{abstract}
The harmonic index of a graph $G$ is defined as $H(G)=\sum_{u v E(G)} \frac{2}{d(u)+d(v)}$, where $d(u)$ denotes the degree of a vertex $u$ in $G$. In this work, we give another expression for the Harmonic index. Using this expression, we give the minimum value of the harmonic index for any triangle-free graphs with order $n$ and minimum degree $\delta \geq k$ for $k \leq n / 2$ and show the corresponding extremal graph is the complete graph $K_{k, n-k}$.
\end{abstract}

Keywords: Harmonic Index; Minimum Degree; Triangle-Free

\section{Introduction}

All graphs considered in the following will be simple. Let $G$ be a graph with vertex set $V(G)$ and edge set $E(G)$. The order and size of graph $G$ are the number of its vertices and number of its edges, respectively. For undefined terminology and notations, we refer the reader to [1].

For a graph $G$, the harmonic index $H(G)$ is defined as

$$
H(G)=\sum_{u v \in E(G)} \frac{2}{d(u)+d(v)} .
$$

It has been found that the harmonic index, which is a special case of general sum-connectivity index, correlates well with the Randic index [2,3] and the $\pi$-electronic energy of benzenoid hydrocarbons [4,5]. In [6], Favaron et al. considered the relation between harmonic index and the eigenvalues of graphs. Zhong [7] found the minimum and maximum values of the harmonic index for connected graphs and trees, and characterized the corresponding extremal graphs. Recently, Wu et al. [8] give a best possible lower bound for the harmonic index of a graph (a triangle-free graph, respectively) with order $n$ and minimum degree at least two and characterize the extremal graphs. In this work, we will give a best

\footnotetext{
*Research supported by the National Natural Science Foundation of China (No.11101097) and Foundation for Distinguished Young Talents in Higher Education of Guangdong, China (No.LYM11061).
}

possible lower bound for the harmonic index of a triangle-free graph with order $n$ and minimum degree at least $k$. We show the corresponding extremal graph is the complete bipartite graph $K_{k, n-k}$.

\section{Another Expression for the Harmonic Index}

Before we go forwards to investigate the relationship between the Harmonic index and the minimum degree $\delta(G)$ of triangle-free graphs, we will give another expression for the Harmonic index in this section, which is vital in sequel.

Let $G$ be a graphs with order $n$ and minimum degree $\delta(G) \geq k$. Denote by $x_{i, j}\left(x_{i, j} \geq 0\right)$, the number of edges joining the vertices of degrees $i$ and $j$. Denote by $n_{i}$ the number of vertices of degree of $i$. Then

$$
\begin{aligned}
& H(G)=\sum_{k \leq i \leq j \leq n-1} \frac{2}{i+j} x_{i, j} \\
& n_{k}+n_{k+1}+\cdots+n_{n-1}=n .
\end{aligned}
$$

By counting the edges that incident to a vertex of degree $i, i=k, \cdots, n-1$, one obtains

$$
\sum_{\substack{j=k \\ j \neq i}}^{n-1} x_{i, j}+2 x_{i, i}=i n_{i} \text {, i.e. } n_{i}=\frac{1}{i}\left(\sum_{\substack{j=k \\ j \neq i}}^{n-1} x_{i, j}+2 x_{i, i}\right) \text {. }
$$

Substituting Equation (3) back into Equation (2) and performing appropriate rearrangements, we get 


$$
\sum_{k \leq i<j \leq n-1}\left(\frac{1}{i}+\frac{1}{j}\right) x_{i, j}+2 \sum_{i=k}^{n-1} \frac{x_{i, i}}{i}=n .
$$

Now, rewriting Equation (1) as

$$
H=\sum_{k \leq i<j \leq n-1} \frac{2}{i+j} x_{i, j}+\sum_{i=k}^{n-1} \frac{x_{i, i}}{i}
$$

and combining Equations (4) and (5) so as to eliminate the term $\sum\left(x_{i i} / i\right)$, we arrive at

$$
n-2 H=\sum_{k \leq i<j \leq n-1}\left(\frac{1}{i}+\frac{1}{j}-\frac{4}{i+j}\right) x_{i, j}=\sum_{k \leq i<j \leq n-1} \frac{(i-j)^{2}}{i j(i+j)} x_{i, j}
$$

i.e.,

$$
\begin{aligned}
H & =\frac{n}{2}-\frac{1}{2} \sum_{k \leq i<j \leq n-1}\left(\frac{1}{i}+\frac{1}{j}-\frac{4}{i+j}\right) x_{i, j} \\
& =\frac{n}{2}-\frac{1}{2} \sum_{k \leq i<j \leq n-1} \frac{(i-j)^{2}}{i j(i+j)} x_{i, j}
\end{aligned}
$$

Remark 2.1 From (7), we see that $H(G) \leq \frac{n}{2}$ for $n$-vertex graph $G$ and the equality holds if and only if $G$ is regular.

\section{Main Results}

First, we give a lower bound for any triangle-free graph with order $n$ and size $m$.

Lemma 3.1 For any triangle-free graph $G$ with order $n$ and size $m$, then

$$
H(G) \geq \frac{2 m}{n},
$$

where equality holds if and only if $m$ is of the form $m=p(n-p)$ for some natural numbers $p$, and $G \cong K_{p, n-p}$.

Proof. For any edge $u v$ of $G$, we have $d(u)+d(v) \leq n$, or it would contain triangle(s). By (1), we have

$$
H(G)=\sum_{u v E(G)} \frac{2}{d(u)+d(v)} \geq \sum_{u v E(G)} \frac{2}{n}=\frac{2 m}{n},
$$

equality holds if and only if $d(u)+d(v)=n$ for every $u v \in E(G)$. Thus, if we denote $d(u)=p$ for an edge $u v$, then each of the $p$ neighbors, including $v$, of $u$ should has degree $n-p$. Similarly, each of the $n-p$ neighbors of $v$ has degree $p$. Therefore, $m=p(n-p)$ and $G \cong K_{k, n-k}$.

Lemma 3.2 Let $w(i, j)=\frac{1}{i}+\frac{1}{j}-\frac{4}{i+j}$, for $0<i<j$, then $w(i, j)$ is decreasing in $i$ and increasing in $j$. Proof. We have

$$
\begin{aligned}
& \frac{\partial w(i, j)}{\partial i}=-\frac{1}{i^{2}}+\frac{4}{(i+j)^{2}}=\frac{(3 i+j)(i-j)}{i^{2}(i+j)^{2}}<0 \text { and } \\
& \frac{\partial w(i, j)}{\partial j}=-\frac{1}{j^{2}}+\frac{4}{(i+j)^{2}}=\frac{(i+3 j)(j-i)}{j^{2}(i+j)^{2}}>0 \text { for } \\
& i<j .
\end{aligned}
$$

Theorem 3.3 Let $G$ be a triangle-free graph with order $n$ and the minimum degree $\delta(G) \geq k$ $(k \leq n / 2)$. Then

$$
H(G) \geq \frac{2 k(n-k)}{n},
$$

where equality holds if and only if $G \cong K_{k, n-k}$.

Proof. Assume $m$ is the size of graph $G$. We divide the proof into the following two cases.

Case 1. $m>k(n-k)$. The result follows by Lemma 3.1.

Case 2. $m \leq k(n-k)$. Note that the maximum degree $\Delta(G) \leq n-k$ for graph $G$, or it would contain triangle(s). By (6) and Lemma 3.2, we have

$$
\begin{aligned}
H(G) & =\frac{n}{2}-\frac{1}{2} \sum_{k \leq i<j \leq n-1}\left(\frac{1}{i}+\frac{1}{j}-\frac{4}{i+j}\right) x_{i, j} \\
& =\frac{n}{2}-\frac{1}{2} \sum_{k \leq i<j \leq n-k}\left(\frac{1}{i}+\frac{1}{j}-\frac{4}{i+j}\right) x_{i, j} \\
& \geq \frac{n}{2}-\frac{1}{2} \sum_{k \leq i<j \leq n-k}\left(\frac{1}{k}+\frac{1}{n-k}-\frac{4}{n}\right) x_{i, j} \\
& =\frac{n}{2}-\frac{1}{2}\left(\frac{1}{k}+\frac{1}{n-k}-\frac{4}{n}\right) \sum_{k \leq i<j \leq n-k} x_{i, j} \\
& \geq \frac{n}{2}-\frac{m}{2}\left(\frac{1}{k}+\frac{1}{n-k}-\frac{4}{n}\right) \\
& \geq \frac{n}{2}-\frac{k(n-k)}{2}\left(\frac{1}{k}+\frac{1}{n-k}-\frac{4}{n}\right) \\
& =\frac{2 k(n-k)}{n} .
\end{aligned}
$$

For equalities to hold above, we must have $m=k(n-k)=x_{k, n-k}$, which means that $G \cong K_{k, n-k}$.

\section{REFERENCES}

[1] J. A. Bondy and U. S. R. Murty, "Graph Theory," Springer, 2008. doi:10.1007/978-1-84628-970-5

[2] X. Li and I. Gutman, "Mathematical Aspects of Randic'Type Molecular Structure Descriptors," Mathematical Chemistry Monographs, Vol. 1, Kragujevac, 2006.

[3] X. Li and Y. T. Shi, "A Survey on the Randić Index," MATCH: Communications in Mathematical and in Computer Chemistry, Vol. 59, No. 1, 2008, pp. 127-156.

[4] B. Lučić, S. Nikolić, N. Trinajstić, B. Zhou and S. I. Turk, "Sum-Connectivity Index," In: I. Gutman and B. Furtula, Eds., Novel Molecular Structure Descriptors-Theory and 
Applications I, University of Kragujevac, Kragujevac, 2010, pp. 101-136.

[5] B. Lučić, N. Trinajstić and B. Zhou, "Comparison between the Sum-Connectivity Index and Product-Connectivity Index for Benzenoid Hydrocarbons," Chemical Physics Letters, Vol. 475, No. 1-3, 2009, pp. 146-148. doi:10.1016/j.cplett.2009.05.022

[6] O. Favaron, M. Mahó and J. F. Saclé, "Some Eigenvalue Properties in Graphs (Conjectures of Graffiti-II)," Dis- crete Mathematics, Vol. 111, No. 1-3, 1993, pp. 197-220. doi:10.1016/0012-365X(93)90156-N

[7] L. Zhong, "The Harmonic Index for Graphs," Applied Mathematics Letters, Vol. 25, No. 3, 2012, pp. 561-566. doi:10.1016/j.aml.2011.09.059

[8] R. Wu, Z. Tang and H. Deng, "A Lower Bound for the Harmonic Index of a Graph with Minimum Degree at Least Two," Filomat, Vol. 27, No. 1, 2013, pp. 51-55. 